\title{
Fortbildung just in time
}

\section{Fachlicher Austausch - Expertenrat}

Das neue DentalSchool Forum - ein fachliches Netzwerk für approbierte Zahnärzte - gibt in der Praxis wichtige Entscheidungshilfen. Die Teilnahme am Informationsaustausch erfolgt über Smartphone, Tablet oder PC. In einer geschlossenen Gruppe können Textnachrichten und Bildinformationen ausgetauscht und gemeinsam kommentiert werden. Grundlage ist die neue DentalSchool ForumApp, die von Appleund Android-Smartphone-Betriebssystemen unterstützt wird und auch im Internet genutzt werden kann. Stellen Sie Ihre Fragen ans Forum, schildern Sie Ihre Fälle, Probleme und Erfahrungen. Verfolgen und profitieren Sie von den fachlichen Fragen und Erfahrungen anderer Kollegen - deutschlandweit. Zu einem aktuellen Patienten, zur Praxisorganisation, zu Investitionen, Verfahren und Werkstoffen, Hygiene, Praxisbegehung - alles dreht sich ums Fachliche. Antworten kommen rasch aus allen Ecken Deutschlands. Fachlicher Mentor ist Univ.-Prof. Dr. Rainer Hahn, der die Fragen zeitnah beantwortet und Beiträge moderiert. Das Netzwerk wurde 2015 als WhatsApp-Gruppe gegründet und ist regelrecht explodiert.

Die monatliche Mitgliedsgebühr beträgt lediglich 49,- $€$ (zzgl. MwSt.). Die Mitgliedschaft im 1. Monat ist kostenlos und kann jederzeit gekündigt werden. Danach läuft eine Mitgliedsperiode jeweils 6 Monate. DentalSchool StudyClub-Mitglieder nutzen das Forum kostenlos. Datenschutz wird groß geschrieben. Weitere Infos zum DentalSchool Forum finden Sie unter: www.dentalschool.de/Forum.

Nach einer Pressemitteilung der Cumdente $\mathrm{GmbH}$, Tübingen 